\title{
Evaluation of the Prescription Pattern of Antimicrobial Agents in Hospitalised Paediatric Patients in a Tertiary Care Hospital of Kathmandu, Nepal
}

\author{
Khadka A', Rayamajhi $\mathrm{H}^{2}$, Shrestha $\mathrm{S}^{3}$
}

\section{Abstract}

Introduction: Infectious diseases are one of the most frequent reasons for hospital admission for children and antimicrobials are commonly used to treat them. There is higher prevalence of irrational prescriptions of antimicrobials in such population. This study was conducted to survey the pattern of antimicrobial prescription in Paediatric inpatient.

Material and Methods: This was retrospective, descriptive, observational study. The data was collected from prescription records. The paediatric population included in the study was categorised into three age groups. A total of 87 prescriptions were studied and the information was recorded in performa. Data were recorded in MS Excel and Graph Pad Prism version 6 was used for analysis.

Results: The number of male child was outnumbered by female child. The average age of the patient on antimicrobial prescription was $5.34 \pm 2.18$ years. The mean duration of hospital stay was $6.17 \pm 2.01$ days. The common indications for antimicrobial prescriptions were upper respiratory tract infections, diarrhoeal illness, urinary tract infections, enteric fever, etc. The antimicrobials prescription rate was 1.33. Oral route was found to be commonly used than parenteral route. The generic prescription rate was $33.6 \%$. The most common drug prescribed was ceftriaxone followed by amoxicillin + clavulanic acid, ciprofloxacin, metronidazole and amikacin. The $62.5 \%$ of antimicrobials were prescribed from National Essential List of Medicines.

Conclusion: This study highlights the prescription pattern of antimicrobial agents in hospitalised Paediatric population in a tertiary care hospital.

Key words: antimicrobials; amoxicillin-clavulanic acid; ceftriaxone; children; diseases; prescription

\section{INTRODUCTION}

nfections are common in children and are responsible for a large Inumber of hospital admissions. ${ }^{1}$ Antimicrobials are frequently used to treat infections. Unfortunately, a great number of antimicrobial prescriptions are unnecessary or inappropriate. ${ }^{2}$ Antimicrobials are unnecessarily prescribed for viral infections such as common cold and
${ }^{1}$ Dr. Anjan Khadka, MBBS, MD, Assistant Professor; ${ }^{2} \mathrm{Dr}$. Helena Rayamajhi, BDS, MD, Assistant Professor; Both from Department of Pharmacology, Nepalese Army Institute of Health Sciences, College of Medicine, Kathmandu, Nepal; ${ }^{3}$ Dr. Shova Shrestha, MBBS, MD, Lecturer, Department of Paediatrics, Patan Academy of Health Sciences, Lagankhel, Lalitpur, Nepal.

\author{
Address for correspondence \\ Dr. Anjan Khadka, \\ Lecturer, \\ Department of Pharmacology, \\ Nepalese Army Institute of Health Sciences, \\ College of Medicine, \\ Kathmandu, Nepal \\ Tel No: +977-9841231875 \\ E-mail: anjankhadka14@gmail.com
}

Acknowledgements: All the patients whose prescriptions were included and the staffs of Paediatric ward.

Funding: Nil

Conflict of Interest: None

Permission from IRB: Not Submitted

\section{How to cite}

Khadka A, Rayamajhi H, Shrestha S. Evaluation of the Prescription Pattern of Antimicrobial Agents in Hospitalised Paediatric Patients in a Tertiary Care Hospital of Kathmandu, Nepal. J Nepal Paediatr Soc 2018;38(3):157-62.

doi: http://dx.doi.org/10.3126/jnps.v38i3.26518

Submitted on: 2019-12-01

Accepted on: 2020-02-12

This work is licensed under a Creative Commons Attribution 3.0 License. 
non-infectious diarrhoea. Similarly, many physicians are prescribing antimicrobials as empiric therapy without considering the risk and necessity. Such rampant prescription of antimicrobials often results in increased incidence of adverse drug reactions, treatment failure, poly-pharmacy, drug interactions, increased healthcare costs and moreover the burning problem of the century i.e. antimicrobial resistance. ${ }^{3}$ It may be due to factors such as tendency to cure early and easily, knee jerk response to prescribe drugs for every symptoms, following the general trend of the colleagues and luring marketing strategies of pharmaceutical companies.

Prescription pattern study helps in identifying irrational prescribing such as poly-pharmacy, over prescription, wrong prescription, etc. It is also essential for prescriber, hospital and patient to know the trends, compliance and adherence to treatment guidelines. The development of antimicrobials prescribing guidelines for children is crucial and need of time.

The prevalence of antimicrobial prescription in our region is $91 \%$ whereas in India, Italy, United Kingdom and Sierra Leone are $91.6 \%, 52 \%, 14 \%$ and $83 \%$ respectively. ${ }^{4-7}$ Though the literature regarding the antimicrobials use in paediatric population in our setting is extensive, there is lack of regulations to monitor the antimicrobials prescription based on standard treatment guidelines. ${ }^{8-9}$ Similarly, culture and sensitivity data to guide antimicrobial usage is also lacking in our part of the world.${ }^{10}$ There is need of studies to obtain information about prescribing habits so as to determine the various lacunae observed to improve prescribing in children. The objective of the study was to evaluate the prescription pattern of antimicrobial agents in hospitalised Paediatric population in a tertiary care hospital of Kathmandu.

\section{Material and Methods}

This was retrospective, descriptive, observational type of study of three months duration carried out from July 2019 to September 2019 in Department of Paediatric of a tertiary care hospital after getting approval from Institutional Ethics Committee. The data was collected from prescription by multiple paediatricians that were available in inpatient record section of Paediatric Department. Those prescriptions which contained the child particulars, diagnosis, prescription with drug name, dose, durations, frequency, route of administration, date of admission and discharge along with the signature of the prescriber were only included. Among such records, only the prescription of patients aged from one month to 12 years including both genders who were admitted in Paediatric ward and prescribed one or more antimicrobials during their admission were included. The prescriptions for the patients less than one month (i.e. neonates) and more than 12 years (i.e. adolescents and adults) were excluded from the study. The Paediatric patients included in the study were categorised into four groups i.e. one month to one year (infant), one year to three years (toddler), three years to five years (preschooler) and five years to 12 years (school aged child). ${ }^{11}$

Out of 290 prescriptions, only 87 prescriptions met the inclusion criteria and were included. The information regarding the patient's particulars, diagnosis, antimicrobials used, duration of treatment and length of hospital stay were recorded in performa. Further, the average number of antimicrobials per prescription, route of administration, group of antimicrobials prescribed, numbers of drugs prescribed in generic name were also evaluated. The recorded information was also compared among four groups of Paediatric patients. Data were recorded in MS Excel and statistical analysis was done using Graph Pad Prism version 6. The results were expressed as percentage or as mean \pm standard deviation (SD).

\section{Results}

The number of prescriptions studied was 87 (30 from age group one month to one year, 19 from age group one to three years, 11 from age group three years to five years and 27 from age group five to 12 years). The total number of male children and female child were 41 and 46 respectively. The sex distribution in each groups have been shown in table 1 . The average age of the patient on antimicrobial prescription was $5.34 \pm 2.18$ years. The mean duration of hospital stay was $6.17 \pm$ 2.01 days $(6.60 \pm 2.18$ days in age group one month to one year, $6.22 \pm 2.20$ days in age group one to five years and $5.60 \pm 1.29$ days in age group five to 12 years).

The indications for antimicrobial prescriptions were upper respiratory tract infections, diarrhoeal illness, urinary tract infections, enteric fever, pneumonia, febrile seizures, pyrexia of unknown origin, pleural effusion, meningitis, nephrotic syndrome and others like otitis, acute rheumatic fever, constrictive pericarditis, wound infection, etc. (Table 2) The common diseases seen in children of age group one month to one year was upper respiratory tract infections $(23.3 \%)$ followed by diarrhoeal illnesses $(16.7 \%)$, urinary tract infections $(13.3 \%)$ and enteric fever (13.3\%). In age group one year to three years, the common diseases were upper respiratory tract infections $(21 \%)$, urinary tract infections $(21 \%)$, diarrhoeal illnesses $(15.7 \%)$, and pneumonia (10.5\%). In age group three years to five years, the common diseases were diarrhoeal illnesses (36.3\%), upper respiratory tract infections (18.1\%) and pneumonia $(18.1 \%)$. Similarly, in age group five years to 
12 years, the common diseases were upper respiratory tract infections $(44.4 \%)$ followed by diarrhoeal illnesses (18.5\%) and enteric fever (11.1\%).

The total antimicrobials prescribed were 116 from seven drug groups. The average number of antimicrobials per prescription was 1.33 with a range of minimum one antimicrobial to three antimicrobials per patient. Out of 116 antimicrobials prescribed, 65 were prescribed for oral administration and 51 for parenteral administration. The number of children of age group one month to one year, one year to three years, three years to five years and five years to 12 years who were administered drug orally were 22,14 , nine and 20 respectively whereas via parenteral route was 10 , nine, six and 26 respectively. The number of antimicrobials prescribed with their generic name in age group one month to one year, one year to three years, three years to five years and five

Table 1: Baseline characteristics

\begin{tabular}{lccc}
\hline Age groups & Male & Female & Total \\
\hline One month - one year & 18 & 12 & 30 \\
\hline One year - three years & 7 & 12 & 19 \\
\hline Three years - five years & 5 & 6 & 11 \\
\hline Five years - 12 years & 11 & 16 & 27 \\
\hline Total & $\mathbf{4 1}$ & $\mathbf{4 6}$ & $\mathbf{8 7}$ \\
\hline
\end{tabular}

years to 12 years were 11, eight, four and 16 (total 39) respectively whereas with their trade name in age group one month to one year, one year to three years, three years to five years and five years to 12 years were 21 , 15,11 and 30 (total 77 ) respectively.

The pattern of prescription of antimicrobials is depicted in figure 1. Among prescribed groups, the most common drug prescribed was ceftriaxone followed by amoxicillin + clavulanic acid, ciprofloxacin, metronidazole, amikacin and ofloxacin. The other drugs prescribed were azithromycin, levofloxacin, cefotaxime + sulbactam, cefexime, tinidazole, cephalexin, ampicillin, vancomycin, gentamycin and ceftazidime. The number of antimicrobials used in age group one month to one year, one year to three years, three years to five years and five years to 12 years were $27.6 \%, 19.8 \%, 12.9 \%$ and $39.6 \%$ respectively.

The number of prescriptions containing single antimicrobial was $55(63.2 \%)$ and more than one antimicrobial was 32 (36.8\%). The commonly prescribed fixed drug combinations were amoxicillin + clavulanic acid $(16.4 \%)$ and cefotaxime + sulbactam (5.2\%). Out of 16 different antimicrobials prescribed, 10 were from National List of Essential Medicines Nepal (Fifth Revision) 2016. ${ }^{12}$

Table 2: Pattern of diseases as indications of antimicrobials

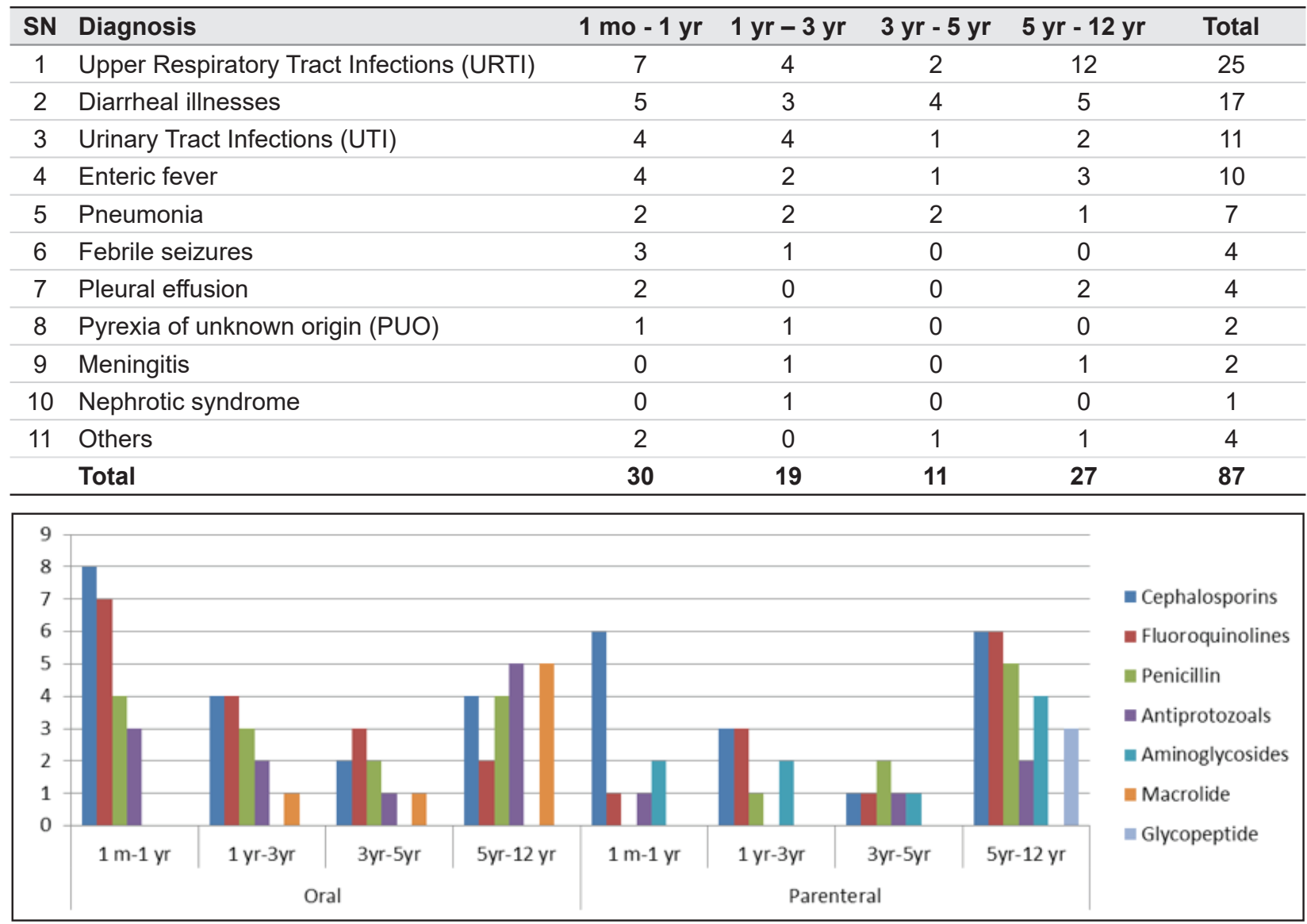

Fig. 1: Pattern of prescription of antimicrobials 


\section{Discussion}

This study was focused on the analysing prevailing disease pattern and antimicrobials use for various infections in admitted patients in Paediatric ward within the duration of three months. The findings of this study provide the information regarding the indications and use of common antimicrobial practice in Paediatric population. Female $(52.9 \%)$ paediatric patients were higher in number than males $(47.1 \%)$ which are in contrast to the study conducted in Kathmandu, Nepal (Male 58.5\%, female 41.5\%) and Guwahati, India (Male $58.3 \%$, female $41.7 \%) .{ }^{4,13}$

Upper respiratory tract infection was most common in age group one month to one year and five years to 12 years whereas urinary tract infection and diarrhoeal illness were common in age group one year to three years and age group three years to five years respectively. This finding is similar to the study done in Guwahati, India but different than the study conducted in Nagpur, India which showed acute gastroenteritis as common diagnosis in one month to one year and five years to 12 years age group whereas lower respiratory tract infection in one year to five years age group. ${ }^{13,14}$

The generic prescribing rate of antimicrobials $(33.6 \%)$ was less than prescribing with trade name $(66.4 \%)$ in this study. Generic prescription rate was found to be more than that of study conducted in Andhra Pradesh, India (23.43\%) and in North East India (8.2\%) but less than similar type of study conducted in Punjab, India (58\%), Sudan (49.3\%) and Nigeria (68.9\%). . $^{6,15-18}$ Generic prescribing has been found to reduce the cost of medicines and also promote better communication among health care providers. This study found that oral route $(56 \%)$ of administration was common than parenteral route $(44 \%)$ which is contrast to the study published by Palikhe $\mathrm{N}$ et al. (75\% parenteral, $25 \%$ oral), Kanish et al. ( $92 \%$ parenteral, $8 \%$ oral), Dutta et al. (75\% parenteral) and Laya VR et al. (87.7\% parenteral, $12.3 \%$ oral).4,6,16,19 This may be due to the less severity of illness, better compliance of child, lower cost of therapy and knowledge of physicians regarding the hazards of injections. WHO also recommends for lowering the use of parenteral drugs as it reduces the cost and risks. ${ }^{13}$

The most frequently prescribed top three antimicrobials among all prescription were ceftriaxone (Third generation cephalosporin, 17.2\%) followed by combination of amoxicillin and clavulanic acid (extended spectrum amino-penicillin + beta lactamase inhibitor, 16.8\%) and ciprofloxacin (first generation fluoroquinolones, $12.9 \%$ ). This finding differs with findings of Kanish et al which showed amikacin (87\%) among aminoglycosides, amoxicillin plus clavulanic acid (20\%) among beta lactams and ofloxacin $(52.5 \%)$ among fluoroquinolones as mostly prescribed antimicrobials. ${ }^{6}$ The studies done Palikhe $\mathrm{N}$ and Dutta $S$ et al. demonstrated ceftriaxone as commonest antimicrobials prescribed which is similar to our study. ${ }^{4,16}$

Upper respiratory tract infections (28.7\%) were the most common diagnosis among all patients prescribed with antimicrobials which is in congruent to the study done by Pradeep Kumar et al. and Jangra et al. ${ }^{15,20}$ Studies done by Jangra et al and Bhanwra et al. showed that upper respiratory infections were treated with antimicrobials though most of them were of viral origin which might be similar in our findings too. ${ }^{20,21}$ Duration of hospital stay $(6.17 \pm 2.01$ days $)$ was found similar to study conducted by Choudhary et al. (6.05 \pm 3.45 days) and more than the study done by Heys et al. (1.8 days). ${ }^{13,22}$ Though, antimicrobial agents are commonly used for the treatment of paediatric illnesses like URTI, fever, UTI, infectious diarrheas, etc the presumed indications was taken from the working diagnosis as documented in the medical record instead of applying culture sensitivity reports as the basis of prescriptions. Antimicrobials prescriptions were used maximum in age groups of five to 12 years which is similar to the study done in the Madhya Pradesh, India. ${ }^{23}$

The average number of antimicrobials per prescription was less than two which is as per recommendation of $\mathrm{WHO} .{ }^{24}$ The $62.5 \%$ of antimicrobials prescribed were from National List of Essential Medicines which is less than that of study conducted by Pise et al. (77.5\%) and Kanish et al (86\%). ${ }^{6,12,25}$ The prescription from essential drug list need to be promoted and availability of essential drug list to be maintained in ward.

Paediatric patients admitted in ward were commonly prescribed multiple antimicrobials. Most of the prescriptions were found to be empiric and broad spectrum based on physician prior experience rather than culture sensitivity proven. It was found that culture reports were not examined before initiating the antimicrobials in most of the cases. This led to overuse or misuse of antimicrobials, increased the burden of antimicrobial resistance, exposed the patients to adverse effects and increased treatment cost. ${ }^{26}$

There is need for strict surveillance, stringent infection control practices as well as rational prescription of antimicrobials. Education of the prescriber on antimicrobial stewardship and rational prescribing will improve rational prescribing of antimicrobials to a greater extent. Unnecessary antimicrobial prescriptions for viral 
diseases, and choosing second choice therapies for certain infective conditions, can increase antimicrobial resistance in paediatric population from their early life ${ }^{26}$ The findings of this study are expected to provide relevant information to paediatricians, general practitioners and those involved in antimicrobial stewardship.

Study was conducted in single centre with small sample. The prescriptions were selected from shorter duration and only of admitted patients aged one month to 12 years were analysed. This study was descriptive and underlying reasons and factors for prescription by multiple prescribers were not evaluated. The cost benefits, patient's knowledge of correct dosage, adverse drug reactions, drug interactions and antimicrobial resistance pattern were not assessed.

\section{Conclusion}

This study highlights the contemporaneous insights into the practice of antimicrobials in paediatric inpatients. The average prescription rate of antimicrobials was not high. The most commonly used antimicrobials were beta lactams and diagnosis was URTI. The common route of administration was oral. The maximum antimicrobial prescription was found in age group five years to 12 years. Generic prescribing and selection of antimicrobials from essential medicine list were lesser than the contemporary studies. Hence, this study will complement and show the way forward for the further study with larger sample size as there is necessity of drafting of standard treatment guidelines as per local prevalence and update the antimicrobial policy periodically along with implementation of antibiotic stewardship in the hospitals.

\section{References}

1. Baidya S, Hazra A, Datta S, Das AK. A study of antimicrobial use in children admitted to Pediatric medicine ward of a tertiary care hospital. Indian J Pharmacol. 2017; 49(1):10-15. DOI:10.4103/02537613.201034

2. Principi N, Esposito S. Antimicrobial stewardship in Pediatrics. BMC Infect Dis. 2016;16:424. DOI:10.1186/ s12879-016-1772-z

3. Choudhury R, Panda S, Singh DV. Emergence and dissemination of antimicrobial resistance: A global problem. Indian J Med Microbiol. 2012;30:384-90. DOI: 10.4103/0255-0857.103756

4. Palikhe N. Prescribing pattern of antimicrobials in Pediatric hospitals in Kathmandu valley. Kathmandu Univ Med J. 2004;2:6-12. PMID: 19780281

5. Clavenna A, Bonati M. Differences in antimicrobial prescribing in Pediatric outpatients. Arch Dis Child. 2011;96(6):590-5. DOI: 10.1136/adc.2010.183541

6. Kanish R, Gupta K, Juneja S, Bains HS, Kaushal S. Prescribing pattern of antimicrobials in the department of Pediatrics in a tertiary care medical college hospital in Northern India. Asian Journal of Medical Sciences. 2014;5 (4):69-72. DOI: 10.3126/ajms.v5i4.10213

7. Cole CP, James PB, Kargbo AT. An evaluation of the prescribing patterns for under-five patients at a Tertiary Pediatric Hospital in Sierra Leone. J Basic Clin Pharm. 2015;6:109-14. DOI: 10.4103/0976-0105.168051

8. Upadhyay S, Sharma A, Rijal P, Shrestha S. Review of Pediatrics In-Patient at Nepal Medical College and Teaching Hospital. J Nepal Paedtr Soc. 2011;31(1):2529. DOI: 10.3126/jnps.v31i1.3262

9. Shrestha R, Shrestha JM, Gurung B. Antimicrobial usage and its sensitivity pattern in the NICU.

Kathmandu Univ Med J (KUMJ). 2012;10(38):27-32. DOI: 10.3126/kumj.v10i2.7339

10. Harbarth S, Samore MA. Antimicrobial resistance determinants and future control. Emerg Infect Dis. 2005 Jun; 11(6): 794-801. DOI: 10.3201/eid1106.050167

11. Hai MA. Understanding early childhood- A look. Int J Acad Res. 2017;1(1):105-8.

12. National List of Essential Medicines Nepal (Fifth Revision). Government of Nepal. Ministry Of Health, Department Of Drug Administration. 2016. Available from: http://apps.who.int/medicinedocs/documents/ s23537en/s23537en.pdf

13. Choudhury DK, Bezbaruah BK. Antimicrobial Prescriptions Pattern in Paediatric In-Patient Department Gauhati Medical College and Hospital, Guwahati. J App Pharm Sci. 2013;3(08):144-8. DOI: 10.7324/JAPS.2013.3825

14. Deshmukh SN, Mahajan MM. A study of prescription pattern of antimicrobials in Pediatric in-patients at a tertiary care hospital in central India. IJPR. 2016;6(8):286-90. DOI: 10.7439/ijpr.v6i8.3518

15. Pradeepkumar B, Alameri T, Narayana G, Reddy YP, Ramaiah JD. Assessment of antimicrobial prescribing pattern in Pediatric patients: A cross-sectional hospitalbased survey. CHRISMED J Health Res. 2017;4:2357. DOI: 10.4103/cjhr.cjhr_52_17

16. Dutta S, Bhattacharjee A, Devi MN. Prescription pattern of antimicrobials in Paediatric inpatients at a tertiary care hospital in North East India. Int J Basic ClinPharmacol. 2017;6:2384-7. DOI:0.18203/23192003.ijbcp20174364

17. Ahmed AM, Awad Al. Drug use practices at Pediatric hospitals of Khartoum State, Sudan. Ann Pharmacother 2010;44:1986-93. DOI:10.1345/aph.1P423. 
18. Fadare $\mathrm{J}$, Olatunya $\mathrm{O}$, Oluwayemi $\mathrm{O}$, Ogundare $\mathrm{O}$. Drug prescribing pattern for under-fives in a Pediatric clinic in South-Western Nigeria. Ethiop J Health Sci. 2015;25:73-8. DOI: 10.4314/ejhs.v25i1.10

19. Rad LV, Modupalli A. Prescribing pattern of antimicrobials in Pediatric inpatient department of a tertiary care teaching hospital in Bangalore. IOSR-JPBS. 2015;10(6):26-32. DOI: 10.9790/300810412632

20. Jangra S, Bhyan B, Chand W, Saji J, Ghoghari R.To assess prescribing pattern of antimicrobials in department of Pediatrics at tertiary care teaching hospital. JDDT. 2019;9(2):192-6. DOI: 10.22270/jddt. v9i2.2402

21. Bhanwra S. A study of non-prescription usage of antimicrobials in the upper respiratory tract infections in the urban population. J Pharmacol Pharmacother. 2013;4(1):62-4. DOI: 10.4103/0976-500X.107687

22. Heys $M$, Rajan $M$, Blair $M$. Length of paediatric inpatient stay, socio-economic status and hospital configuration: a retrospective cohort study. BMC
Health Serv Res. 2017;17(1):274. DOI: 10.1186/ s12913-017-2171-x.

23. Khare S, Purohit $M$, Sharma M, Tamhankar AJ, Lundborg CS, Diwan V, et al. Antibiotic Prescribing by Informal Healthcare Providers for Common Illnesses: A Repeated Cross-Sectional Study in Rural India. Antibiotics. 2019;8(3):139. DOI: 10.3390/ antibiotics8030139

24. Kebede HK, Gesesew HA, Woldehaimanot TE, Goro KK. Antimicrobial use in paediatric patients in a teaching hospital in Ethiopia. PLoS One. 2017;12(3):e0173290. DOI: 10.1371/journal.pone.0173290.

25. Pise HN, Padwal SL, Jadhav RR, Deshmukh VS, Jadhav AD, Kolhe AM. Drug prescribing and dispensing pattern in pediatrics outpatient clinic of a rural tertiary-care teaching hospital. Natl J Physiol Pharm Pharmacol 2015;5:313-7. DOI: 10.5455/ njppp.2015.5.0902201546

26. Lomazzi M, Moore M, Johnson A, Balasegaram M, Borisch B. Antimicrobial resistance-moving forward? BMC public health. 2019;19(1):858. DOI:10.1186/ s12889-019-7173-7 